\title{
Können standardisierte Messverfahren das Gutachterverfahren im Rahmen der ambulanten Psychotherapie verbessern?
}

Das Gutachterverfahren im Rahmen der Beantragung von Richtlinienpsychotherapie war von Beginn an umstritten. Ein Teil der Probleme kommt daher, dass dem Verfahren ein derart großes Spektrum an Funktionen zugeschrieben wird. Eigentlich sind die Psychotherapiegutachten nur ein Wirtschaftlichkeitsprüfverfahren und entbinden Psychotherapeuten von dieser Kontrollmaßnahme, die in der Kassenärztlichen Versorgung vorgesehenen ist. Mit diesem Verfahren lässt sich jedoch kein Geld einsparen, da bei Ablehnung eines Antrags auf Kostenübernahme der freie Therapieplatz sofort mit einem anderen Patienten besetzt wird. Das Gutachterverfahren ist insofern ein sozialmedizinisches Prüfverfahren der Notwendigkeit einer Therapie im Sinne des Sozialrechts, d.h. ein Verfahren der Ressourcenallokation. Es reserviert knappe Therapieplätze für die Patienten, bei denen eine Therapie sinnvoll erscheint, und die nicht anders betreut werden können. Ob das Gutachterverfahren diesen Effekt erfüllt, kann diskutiert werden, wenn man die sehr geringen Ablehnungsquoten und die langen Wartelisten bedenkt. Bleibt noch die Aufgabe der Qualitätssicherung. Sozialrechtlich betrachtet sind Gutachter unter diesem Gesichtspunkt keine Supervisoren, sondern müssen die Zweckmäßigkeit und Spezifität des Therapieplans prüfen, d.h. sicherstellen, dass der Verhaltenstherapeut nach den Regeln der Kunst liefert, wenn die Krankenkasse Verhaltenstherapie "bestellt» und bezahlt. Ob dies erreicht wird, wird ebenfalls in Frage gestellt, mit dem Argument, dass die therapeutischen Berichte, auf denen die Gutachten basieren, zweckdienlich geschönt und realitätsfern seien.

Vor diesem Hintergrund ist eine naheliegende Frage, ob die Gutachten und deren Wirkungen durch den Einsatz von standardisierten Instrumenten und Rückmeldeprozessen verbessert werden können, oder ob dadurch alles nur noch komplizierter würde. In der folgenden Pro-Contra-Diskussion stellen die Autoren dankenswerterweise die zu berücksichtigenden Argumente dafür und dagegen kontrapunktisch einander gegenüber, um dem Leser eine eigene Urteilsbildung zu ermöglichen.

Michael Linden, Berlin 
Die Frage, die hier diskutiert werden soll ist, ob das Gutachterverfahren durch den Einsatz psychometrischer Instrumente sinnvoll ergänzt und optimiert werden kann. Wir plä-

\section{Gutachterverfahren mit Einbezug psychometrischer Informationen}

In der Bundesrepublik Deutschland existiert eines der besten psychotherapeutischen Versorgungssysteme weltweit. Psychotherapie ist als gesetzliche Krankenversicherungsleistung für alle behandlungsbedürftigen Patienten, unabhängig von ihrer finanziellen Situation, zugänglich. Darüber hinaus liegt nachweislich ein hoher Qualitätsstandard vor. Vorliegende Untersuchungen sprechen für eine hohe Wirksamkeit mit Effektgrößen, die im Bereich meta-analytischer Vergleichsuntersuchungen liegen.

Das Gutachterverfahren hat seit Einführung der ambulanten Psychotherapie in die kassenärztliche Versorgung im Jahr 1967 einen wichtigen Beitrag zur Versorgungsqualität in der Bundesrepublik geleistet. Vor dem Hintergrund limitierter Ressourcen im Gesundheitssystem wird im Rahmen des Gutachterverfahrens im Hinblick auf die Leistungspflicht der gesetzlichen Krankenversicherung die Notwendigkeit, Zweckmäßigkeit und Wirtschaftlichkeit einer psychotherapeutischen Behandlung vorab beurteilt. Ziel ist es, die Kostenübernahme von Psychotherapien an den Nachweis einer entsprechenden Behandlungsbedürftigkeit und einer ausreichend positiven Prognose sowie an das Vorliegen eines für das gewählte Therapieverfahren und die Indikation angemessenen und fachlich gerechtfertigten Behandlungsplans zu knüpfen.

Seit einigen Jahren gerät das Gutachterverfahren jedoch zunehmend stärker in die Kritik. Insbesondere werden die hohen Kosten bei gleichzeitig unklarem Nutzen diskutiert. Es wird davon gesprochen, dass das Gutachterverfahren kaum mehr eine Beurteilung der Notwendigkeit und Zweckmäßigkeit einer Behandlung bei einem bestimmten Patienten, sondern des Formulierungsgeschicks des Therapeuten und seiner Kenntnisse in Bezug auf die Begutachtungskriterien abbildet.

Im Zuge der Debatte zur Qualitätssicherung in der ambulanten Psychotherapie wurde gegenüber dem Gutachterverfahren darüber hinaus eingewendet, dass es in seiner derzeitigen Form, die im Kern aus einer vorab durchgeführten Überprüfung der Zweckmäßigkeit und Wirtschaftlichkeit einer beantragten Psychotherapie durch erfahrene Fachvertreter besteht, ein Qualitätssicherungsinstrument ist, dass insbesondere auf Aspekte der Strukturqualität abzielt, jedoch Aspekte der Prozess- und Ergebnisqualität psychotherapeutischer Behandlungen vernachlässigt. Eine Beurteilung der Qualität des Behandlungsprozesses und der zweckmäßigen Anpassung von Interventionen an den Behandlungsverlauf kann durch das derzeitige Gutachterverfahren nicht abgebildet werden. Das Gutachterverfahren als externes Qualitätssicherungsinstrument sollte deshalb im Idealfall durch Maßnahmen der internen Qualitätssicherung, d.h. durch ein unmittelbares Monitoring des Behandlungsprozesses und den Einbezug der Patientenperspektive, ergänzt werden. dieren für die Beibehaltung des Gutachtersystems, jedoch mit einem ergänzenden Einbezug von Ergebnissen aus psychometrischen Tests bei der Therapiebewilligung wie auch der Behandlungsverlängerung. Psychometrische Informationen, die unmittelbar auf Selbsteinschätzungen des Patienten beruhen, stellen nachgewiesenermaßen eine hilfreiche Ergänzung zu dem klinischen Eindruck des Therapeuten dar und können dazu beitragen, negative oder positive Entwicklungen im Therapieverlauf möglichst frühzeitig zu erkennen und entsprechend in die eine oder andere Richtung darauf zu reagieren.

In dem groß angelegten Modellprojekt der Techniker Krankenkasse (TK) zur Qualitätssicherung in der ambulanten Psychotherapie (TK-Modell) wurde in Deutschland erstmalig ein durch psychometrische Informationen adaptiertes Qualitätssicherungs- und Begutachtungsverfahren im Rahmen des Psychotherapiegenehmigungsverfahrens eingesetzt. In der Studie wurde neben dem traditionellen Gutachterverfahren ein modifiziertes Gutachterverfahren auf seine Umsetzbarkeit hin untersucht, das neben einem komprimierten schriftlichen Antrag zusätzlich psychometrische Informationen vorsah. Im Zeitraum Mai 2005 bis Juni 2010 wurden Daten von insgesamt 1708 Patienten erhoben, die bei einem von 245 an der Studie beteiligten niedergelassenen Psychotherapeuten mit unterschiedlicher therapeutischer Ausrichtung (Verhaltenstherapie, Tiefenpsychologisch fundierte Psychotherapie, Analytische Psychotherapie) in den 3 Modellregionen der Kassenärztlichen Vereinigungen (KVen) Hessen, Südbaden und Westfalen-Lippe ambulant behandelt wurden. Ein modifiziertes Bewilligungsverfahren in der Interventionsgruppe wurde dem traditionellen Gutachterverfahren gemäß PsychotherapieRichtlinien in der Kontrollgruppe gegenübergestellt. Die Basis für das adaptierte Verfahren bildete der Einsatz eines umfassenden Inventars psychometrischer Messinstrumente zur Eingangsdiagnostik sowie zum Monitoring des Therapieverlaufs und der Zielerreichung. Die Empfehlung zur Aufnahme einer Therapie wurde abgegeben, wenn 1) der behandelnde Therapeut für den Patienten mindestens eine Diagnose nach ICD-10 vergeben hatte und 2) der Patient in mindestens einem von 3 zentralen Selbstbeurteilungsinstrumenten einen klinisch auffälligen Wert erreichte. Bei den Selbstbeurteilungsinstrumenten handelte es sich um das «Brief Symptom Inventory» (BSI), das «Inventar für Interpersonale Probleme» (IIP-D) sowie um ein störungsspezifisches Instrument, dass in Abhängigkeit der jeweiligen Diagnose ausgefüllt wurde (z.B. «Beck Depressionsinventar» (BDI)). Bei Zutreffen des genannten Kriteriums wurde von der TK eine Langzeittherapie ohne Begutachtung bewilligt. Nur wenn dieses Kriterium nicht vorlag, kam ein modifiziertes Gutachterverfahren zum Einsatz, das schließlich $\mathrm{zu}$ einer Gutachterempfehlung hinsichtlich der Aufnahme oder der Ablehnung einer Behandlung führte. Darüber hinaus wurde im Modellprojekt der Einfluss von routinemäßigen und 
kontinuierlichen Verlaufsrückmeldungen über den therapeutischen Fortschritt (Feedback) untersucht, weshalb die Therapeuten der Interventionsgruppe im Verlauf der Therapie kontinuierlich Rückmeldungen zum Therapiefortschritt in Form von Veränderungsgrafiken und Kennwerten erhielten. Diese Informationen standen auch den Gutachtern bei Verlängerungsanträgen für ihre Entscheidung zur Verfügung, waren aber ausdrücklich nur als Entscheidungshilfe gedacht und nicht verbindlich.

Erste Ergebnisse des Modellprojekts, welche die Bedeutung von Qualitätssicherungs- und Rückmeldesystemen in der ambulanten Psychotherapie unterstreichen, wurden im letzten Jahr veröffentlicht. Insgesamt 91,9\% $(n=692)$ der Patienten in der Interventionsgruppe erfüllten die oben genannten Bewilligungskriterien; für sie wurde die Bewilligung der Therapie ohne zusätzliches Gutachterverfahren von der TK genehmigt. Die übrigen $8,1 \%(n=61)$ erfüllten die Kriterien nicht; für sie wurde das modifizierte Gutachterverfahren eingeleitet. Bezogen auf die Ergebnisqualität zeigte sich, dass die Behandlungsergebnisse der Interventionsgruppe mit dem modifizierten Bewilligungsverfahren im Vergleich zu den Ergebnissen der Kontrollgruppe mit dem traditionellen Gutachterverfahren gleich gut ausfielen. Darüber hinaus konnte ein Einfluss der Therapieverlaufsrückmeldungen, die auf den psychometrischen Informationen basieren, auf die Behandlungsdauer festgestellt werden. Dieser Effekt zeigte sich nach statistischer Kontrolle der in beiden Bedingungen unterschiedlichen Bewilligungspraxis. Bei der Kontrolle der bewilligten Sitzungen zeigte sich eine leichte Reduktion der durchschnittlichen Gesamtdauer der Therapie in der Interventionsgruppe gegenüber der Kontrollgruppe. Zusätzlich blieben Patienten, die zum ersten Messzeitpunkt eine Verschlechterung der Symptomatik aufwiesen, signifikant länger in der Therapie, während Patienten mit frühen positiven Veränderungen tendenziell kürzere Therapien aufwiesen. Die Umsetzung von routinemäßigen Verlaufsrückmeldungen über den therapeutischen Fortschritt (Feedback) auf der Basis eines kontinuierlichen Verlaufsmonitorings mithilfe psychometrischer Messungen kann als wichtiges Instrument der internen Qualitätssicherung in der ambulanten Psychotherapie, insbesondere im Hinblick auf die Prozess- und Ergebnisqualität, gesehen werden. Im Sinne einer patientenorientierten Psychotherapieforschung wird das Ziel einer Verzahnung von Wissenschaft und Praxis sowie einer adaptiven und an den individuellen Bedürfnissen der Patienten orientierten Behandlungsausrichtung verfolgt. Das kontinuierliche Monitoring des Therapieverlaufs basiert auf veränderungssensitiven, reliablen und vom Umfang her angemessenen Selbstbeurteilungsinstrumenten, wie z.B. dem «Fragebogen zur Evaluation von Psychotherapieverläufen» (FEP) oder dem «Outcome Questionnaire-45»(OQ-45). Nach einer statistischen Auswertung werden die gewonnenen Daten des behandelnden Therapeuten zeitnah anhand von Veränderungsgrafiken und Kennwerten rückgemeldet. Zusätzlich können aus den Thera- pieverlaufsdaten Entscheidungsregeln abgeleitet und den Therapeuten als Handlungsempfehlungen bei klinischen Entscheidungen zur Verfügung gestellt werden. Auf der Basis empirischer Entscheidungsregeln wird der Fortschritt oder Rückschritt eines Patienten im Therapieverlauf relativ zu einer aufgrund verschiedener Prädiktoren vorhergesagten Verlaufskurve für diesen Patienten bewertet. Die Vorhersage für eine patientenspezifische Verlaufskurve basiert auf Verlaufsdaten «ähnlicher» Patienten. Hierfür werden Ausgangsmerkmale von Patienten, die einen bedeutsamen Einfluss auf den Behandlungsverlauf haben, wie z.B. die Ausgangsbelastung hinsichtlich Wohlbefinden und Symptomatik, vorherige Therapieerfahrungen oder die Dauer der Problematik, extrahiert. Im TK-Projekt wurden bei der Beantragung von Therapieverlängerungen, neben den Verlaufsrückmeldungen an die Therapeuten, die Ergebnisse der psychometrischen Messungen zusätzlich den Gutachtern zur Verfügung gestellt. Dies geschah explizit nicht in präskriptiver Form, sondern mit dem Ziel, den Gutachtern eine zusätzliche Informationsquelle über den Therapieverlauf und damit eine empirisch gestützte Entscheidungshilfe anzubieten.

Eine Reihe von Studien konnte mittlerweile zeigen, dass Rückmeldungen zum Therapieverlauf eine hilfreiche Ergänzung zum klinischen Eindruck der Therapeuten darstellen und sich positiv auf das Therapieergebnis auswirken können. Insbesondere, wenn es darum geht, negative Entwicklungen im Therapieverlauf möglichst frühzeitig zu erkennen und in der Behandlung darauf zu reagieren, haben sich Rückmeldungen über den therapeutischen Fortschritt als hilfreich erwiesen. Die Ergebnisse der TK-Studie zeigen, dass der überwiegende Teil der Therapeuten aus der Interventionsgruppe die Verlaufsrückmeldungen für die jeweilige Therapie nutzte und die Ergebnisse mit den Patienten besprach. Vor allem mit Patienten, die einen ungünstigen oder unveränderten Verlauf aufwiesen, wurden die Verlaufsrückmeldungen signifikant häufiger besprochen und es wurde häufiger versucht, die therapeutischen Interventionen entsprechend anzupassen sowie die therapeutische Beziehung zu verbessern. Die Ergebnisse der Feedbackforschung sprechen dafür, dass Fortschrittsrückmeldungen einen wichtigen Beitrag zu einer stärker auf individuelle Patientencharakteristika und Therapieverläufe ausgerichteten therapeutischen Behandlung leisten können und damit ein wichtiges Instrument zur Optimierung der Prozessund Ergebnisqualität darstellen.

Die Ergebnisse unterstreichen außerdem das Potenzial von Rückmeldesystemen für eine effizientere Verteilung psychotherapeutischer Ressourcen. Im Gegensatz zur derzeitigen Bewilligungspraxis von pauschalen Sitzungskontingenten (für welche die empirische Grundlage bislang fehlt) könnte künftig im Sinne eines «individualisierten Stepped-care-Ansatzes» ein flexibleres Vorgehen bei der Ressourcenverteilung möglich werden. Denkbar wäre beispielsweise ein auf empirischen Entscheidungsregeln basierender Vorschlag zur Therapielänge in Abhängigkeit von patientenspezifischen Ausgangs- 
merkmalen zu Therapiebeginn und Informationen zum Therapierverlauf. Demnach könnte zu Therapiebeginn, basierend auf Daten «ähnlicher» Patienten und anhand verschiedener Prädiktoren, die Einfluss auf den Behandlungsverlauf haben (wie z.B. Ausgangsbelastung, Diagnose, vorherige Therapieerfahrungen oder die Dauer der Problematik), eine patientenspezifische Vorhersage zur indizierten Therapielänge abgeleitet werden. Auf Basis der Rückmeldungen zum Therapieverlauf könnten schrittweise entsprechende Anpassungen der Therapielänge erfolgen. Je nach individueller Problemlage benötigen einige Patienten deutlich größere Sitzungskontingente, um ihre Ziele zu erreichen, während für andere Patienten weniger Sitzungen ausreichen, was nicht notwendigerweise bereits zu Therapiebeginn absehbar ist. Entscheidungen zum Sitzungskontingent könnten demnach durch psychometrische Informationen unterstützt werden.

Insgesamt zeigen die Ergebnisse der TK-Studie, dass sich neue und umfangreiche Qualitätssicherungs- und Rückmeldesysteme, die ein adaptives Eingehen auf die individuellen Bedürfnisse von Patienten ermöglichen, in der ambulanten Routineversorgung prinzipiell ein- und umsetzen lassen und ein modifiziertes Bewilligungsverfahren mit diesen verknüpft werden kann. Aus Sicht der Patienten wurde der Einsatz der Rückmeldungen in der TK-Studie als wichtig und hilfreich erlebt und der Zeitaufwand für die Beantwortung der Fragen als vertretbar wahrgenommen. Die Zufriedenheit der beteiligten Patienten war in der Interventionsgruppe mit psychometrischen Erhebungen sogar höher als in der Kontrollgruppe. Bevor jedoch eine Umsetzung in die Routineversorgung empfohlen werden kann, müssen weitere Erfahrungen mit dieser Art von Modellprojekten unter wissenschaftlicher Begleitung gesammelt werden. Das im TK-Projekt umgesetzte Vorgehen ist für die Praxis noch deutlich zu aufwändig und bedarf weiterer Optimierung.

Wichtig ist es uns, an dieser Stelle jedoch zu betonen, dass psychometrische Instrumente selbst keine klinischen Entscheidungen treffen können. Vielmehr müssen die Ergebnisse psychometrischer Ausgangs- und Verlaufsmessungen immer zunächst noch von einem Therapeuten und/oder Gutachter interpretiert sowie durch deren klinische Perspektive ergänzt werden, bevor Indikationsentscheidungen getroffen werden können. Diese Sichtweise wurde von uns zu Beginn der Diskussion um das TK-Modell betont. Psychometrische Instrumente liefern demnach empirisch gestützte Entscheidungshilfen und keine automatisierten Entscheidungen. Wir müssen uns auch darüber im Klaren sein, dass eine Manipulation psychometrischer Daten nicht ausgeschlossen werden kann. Vielmehr ist durchaus vorstellbar, dass mit der Einführung eines psychometrisch gestützten Begutachtungsverfahrens nach und nach bekannt werden würde, wie entsprechende Instrumente auszufüllen sind, damit etwa die Verlängerung oder Bewilligung einer Therapie erreicht werden kann. Deshalb können psychometrische Instrumente kein Ersatz, sondern nur eine Ergänzung für das Gutachtersystem sein.
Zusammenfassend plädieren wir demgemäß nicht dafür, das Gutachterverfahren einzustellen, sondern für eine Ergänzung des Gutachterverfahrens durch psychometrische Instrumente und die kontinuierliche Rückmeldung psychometrisch gewonnener Informationen an die Psychotherapeuten.

Wolfgang Lutz; Luisa Zaunmüller, Trier

\section{Contra}

\section{Modifikation bestehender Instrumente und höhere Selbstverpflichtung der Gutachter}

Nach den Psychotherapierichtlinien dient das Gutachterverfahren dazu, im Vorfeld einer ambulanten psychotherapeutischen Behandlung die Indikation einer solchen zu beurteilen; dies beinhaltet insbesondere eine Krankheitsprüfung sowie die Beurteilung, ob das gewählte Behandlungsverfahren, die beantragte Dauer sowie die ausgewählten einzelnen Therapiemethoden der Erkrankung angemessen und prognostisch ausreichend sind. Dabei handelt es sich um ein sehr aufwändiges Verfahren, das die Therapieeinleitung zeitlich um Wochen verzögert und auch einen Kostenfaktor darstellt. Von daher ist es legitim, immer wieder die Frage zu stellen, ob dieses Verfahren seinen Zweck erfüllt bzw. sein Geld wert ist, und wo Optimierungspotenziale bestehen. Manchmal ergeben derartige Prüfungen aber auch, dass Bestehendes Sinn macht, oder erforderliche Veränderungen in andere Richtungen gehen müssten. Vorweg gesagt gilt nach unserer Meinung, dass es trotz aller Kritik am Gutachterverfahren derzeit kein besseres Instrument gibt, um eine Qualitätssicherung und Behandlungssteuerung in der ambulanten Psychotherapie zu gewährleisten.

Im konkreten Fall soll die Frage diskutiert werden, ob das Gutachterverfahren durch die Nutzung standardisierter Instrumente verbessert oder gar ersetzt werden kann. Von vielen Seiten wird gefordert, vermehrt standardisierte Fragebögen zur Indikationsstellung und zur Verlaufskontrolle einzusetzen, teilweise als Ergänzung, teilweise aber auch als Ersatz für das derzeitige Verfahren. Allerdings bleiben hier viele Fragen unbeantwortet.

Zunächst soll noch einmal in Erinnerung gerufen werden, dass das Gutachterverfahren bereits vor 10 Jahren eine erhebliche Modifikation erfahren hat, indem erfahrene niedergelassene Psychotherapeuten die Möglichkeit bekommen haben, unter Berücksichtigung verschiedener formaler Aspekte, sich für Kurzzeittherapien von der Gutachterpflicht befreien zu lassen. Somit besteht die Gutachterpflicht für Kurzzeittherapien nur noch für neu niedergelassene Kollegen, bei denen unserer Auffassung nach das Gutachterverfahren (auch im Sinne einer Supervision) unter qualitätssichernden Aspekten durch- 
aus Sinn macht. Ein Großteil der niedergelassenen Kollegen unterliegt damit aber de facto, zumindest im Bereich der Kurzzeittherapie, keinerlei Gutachterpflicht. Insofern würde unter ökonomischen Gesichtspunkten die Neuetablierung einer Indikationsüberprüfung über Selbstbeurteilungsskalen einen Rückschritt bedeuten. Aber auch unter psychotherapeutischen Gesichtspunkten kann eine reine Indikationsstellung aufgrund von Selbstbeurteilungsbögen durchaus kritisch gesehen werden. Patienten neigen bei Selbstbeurteilungsbögen grundsätzlich dazu, sich eher höher zu bewerten, auch um ein erwünschtes Ziel zu erreichen. Alle Ärzte und Psychotherapeuten kennen den Effekt, dass Leistungen aus dem Gesundheitswesen oder anderer Sozialleistungsträger hohe funktionale Bedeutung besitzen [Borgart et al., 2007] und es ist wohl naiv ist zu glauben, dass es unter Patienten nicht schnell bekannt werden würde, dass man eine Psychotherapie dann gewährt bekommt, wenn auf dem «Beck Depressionsinventar» (BDI) z.B. 30 Punkte erreicht werden. Im Übrigen sagt ein hoher Score auf Fragebögen noch nichts darüber aus, ob eine geplante Behandlung einem Störungsbild angemessen ist, die geplante Dauer prognostisch erfolgversprechend ist, usw.

Es wird vorgeschlagen, die Ergebnisse psychometrischer Tests bei Entscheidungen über Behandlungsverlängerungen zu berücksichtigen. Hierzu ist anzumerken, dass damit eigentlich eine Selbstverständlichkeit gefordert ist. Jeder Hausarzt, der eine neue Blutdruckmedikation bei seinem Patienten ansetzt, überprüft natürlich regelmäßig, ob das Medikament seine Wirkung entfaltet. So sollte es für jeden Psychotherapeuten selbstverständlich sein, die mit dem Patienten zu Beginn abgesprochenen Therapieziele auf ihre Zielerreichung hin regelmäßig zu überprüfen und den Einsatz der verschiedenen Therapiemethoden $\mathrm{zu}$ evaluieren. $\mathrm{Ob}$ dies mittels standardisierter Fragebögen stattfindet oder durch das klinische Urteil bezogen auf das Therapieziel vorgenommen wird, ist im Einzelfall zu entscheiden; das Vorgehen selbst entspricht aber eigentlich der Sorgfaltspflicht jedes einzelnen behandelnden Therapeuten. Ebenso selbstverständlich sollte es sein, diese Daten und Informationen dem betreffenden Gutachter im Rahmen des Gutachterverfahrens zur Verfügung zu stellen. Leider muss aus unserer gutachterlichen Sicht festgestellt werden, dass eine solche Überprüfung von Therapiezielen und Prognosen nicht der Realität entspricht; hier entstünde ein erster Ansatzpunkt zu Verbesserungen des Gutachterverfahrens: Die Gutachter sollten sich eine höhere Selbstverpflichtung auferlegen, auch solche Aspekte zu überprüfen. Gegebenenfalls müsste in diesem Zusammenhang auch das Psychotherapie-Vereinbarung (PTV-)Formular «Informationsblatt zum Erstellen eines Berichtes...» stringenter formuliert werden. Hierin liegt unseres Erachtens der pragmatischste Weg zu einer Modifikation des Gutachterverfahrens. Unserer gutachterlichen Erfahrung gemäß gibt es im Bereich der ambulanten Psychotherapie durchaus «Wildwuchs», sowohl hinsichtlich des Einsatzes psychotherapeutischer Methoden (hier wird zahlenmäßig eine deutliche Erhöhung von «Eye Movement Desensitization and
Reprocessing» (EMDR) auch bei nicht passenden Störungsbildern beobachtet, dazu passend eine zahlenmäßig deutlich erhöhte Diagnostik einer posttraumatischen Belastungsstörung) als auch - und das ist unserer Auffassung nach sogar das gravierendere Problem - einer fast durchgängig zu beobachtenden Missachtung sozialmedizinischer Aspekte bei der Behandlung. Ein weiterer kritischer Punkt ist etwa die parallele Behandlung von Familienmitgliedern, die nur in den seltensten Fällen von ambulanten Behandlern kritisch diskutiert wird. Diese und sicherlich noch weitere Aspekte sind unserer Meinung nach im Rahmen der bisherigen Verfahrensweisen (Gutachtertagungen) hinreichend aufzuarbeiten. Sie wären z.B. im Sinne einer Checkliste zur Unterstützung bei der Begutachtung ambulanter Psychotherapiefälle relativ zeitnah umzusetzen und würden bei kritischer Würdigung auch zu einer höheren Ablehnungsquote unter inhaltlichen Aspekten führen. Eine solche inhaltlich stringentere Beurteilung von Psychotherapieplanungen würde der leider deutlich zu beobachtenden Tendenz zur «lebenslangen Psychotherapie» («psychotherapy as way of live») entgegenwirken. Zusätzlich wäre in diesem Zusammenhang zu überprüfen, inwieweit ambulante und stationäre Maßnahmen besser verzahnt werden können. Bedauerlicherweise kommt es häufig vor, dass Patienten erst gegen Ende einer 80-stündigen, schon mehrfach verlängerten Psychotherapie einer eigentlich bereits früher indizierten stationären Behandlung zugeführt werden. Eine iatrogene Chronifizierung einer psychischen Erkrankung, die durch eine erfolglose ambulante Psychotherapie möglicherweise bereits stattgefunden hat, lässt sich dann aber nur noch schwer bis gar nicht mehr korrigieren. Derartige Teilaspekte könnten im Rahmen des aktuellen Gutachterverfahrens zeitnah als entscheidungsrelevant etabliert werden und zu einer deutlichen Qualitätsverbesserung und auch Verkürzung ambulanter Psychotherapie führen.

Ein weiterer Aspekt, der für die Indikationsstellung zu einer ambulanten Psychotherapie wichtig sein könnte, ist der sozialmedizinische Status der Patienten. Medizinische Behandlungen jeglicher Art sollten auch immer zum Ziel haben, die Fähigkeit von Patienten zur Teilhabe am beruflichen oder gesellschaftlichen Leben zu verbessern. Deshalb sollte eine ambulante Psychotherapie grundsätzlich darauf abzielen, arbeitsunfähige Patienten wieder arbeits- und leistungsfähig zu machen. Dieser Aspekt kommt inhaltlich in den zumindest uns bekannten Berichten an den Gutachter fast überhaupt nicht vor und spielt für ambulante Psychotherapeuten leider offensichtlich nur eine geringe Rolle, wie wir oben bereits angesprochen haben. Bei der Sozialmedizin handelt es sich um ein für Patienten und Psychotherapeuten gleichermaßen unangenehmes, konflikthaftes und von externen Gratifikationsaspekten überlagertes Thema, das gerne ausgeblendet wird, da hier mit Widerständen seitens der Patienten zu rechnen ist.

Es stellt sich nur die Frage, wie dieser Aspekt im Rahmen der Patientensteuerung berücksichtigt werden soll. Eine vergleichbare Situation ist im Bereich der stationären Versorgung festzustellen; hier haben z.B. Krankenkassen die Mög- 
lichkeit, über den $§ 51.1$ SGB V Patienten bei länger andauernder Arbeitsunfähigkeit eine medizinische Rehabilitation anzubieten. Kostenträger wäre hier die Rentenversicherung, die dann unter Umständen mithilfe eigener Gutachter entscheidet, ob tatsächlich eine entsprechende Behandlung indiziert ist. Eine Rentenversicherung hat es jedoch einfacher, eine ressourcenorientierte Zuweisungsplanung zu gestalten; hier gibt es eine überschaubare Anzahl von Kostenträgern und die angestellten, trägereigenen Gutachter wissen, wie viele Patienten sie einer stationären Behandlung zuführen können (dürfen), um im Rahmen eines bestimmten Budget zu bleiben. Bei ca. 50000 niedergelassenen Psychotherapeuten, mehr als 100 Krankenkassen (die möglicherweise auch ein Interesse daran haben, dass «ihre» Versicherten besser versorgt werden als die Versicherten anderer Kassen) sowie den KVen sind zu viele Beteiligte und Partikularadressen im System, um eine zentrale Steuerung wirklich behandlungsbedürftiger $\mathrm{Pa}$ tienten in die Richtlinienpsychotherapie sicher zu gewährleisten. Das Problem der Ressourcennutzung ist aber ein Problem des gesamten Gesundheitssystems (man betrachte nur die Diskussionen um unnötige Rückenoperationen bei gleichzeitiger sehr langer Wartezeit auf ambulante Behandlungsplätze bei Fachärzten für Orthopädie oder Ähnliches). Die Lösung dieses Problems dem Gutachterverfahren zuzumuten, geht sicherlich an der Realität vorbei.

Zum Schluss soll noch das Problem der Behandlungssteuerung im Rahmen des Gutachterverfahrens angesprochen werden. Dazu sei folgende Überlegung erlaubt: Wissenschaftliche Untersuchungen haben gezeigt, dass es in der Bevölkerung nahezu eine grenzenlose Zahl an fachlich begründbaren potenziellen Indikationen für Psychotherapie gibt. Grundsätzlich können alle psychischen Erkrankungen mit Psychotherapie behandelt werden, von Angsterkrankungen bis hin zu Demenzen. Das bedeutet, dass nach den Daten des Bundes-Gesundheitssurvey ein Drittel der Bevölkerung in psychotherapeutischer Behandlung sein könnte. Des Weiteren gilt, dass alle Menschen mit körperlichen Erkrankungen durchaus auch psychotherapeutisch nutzbringend behandelt werden könnten, von Diabetes über Hypertonus bis hin zu Schmerzerkrankungen. Damit sind mindestens 60 Millionen Deutsche potenziell mit Psychotherapie behandelbar. Zudem kann Psychotherapie auch bei Lebenskrisen zur Anwendung kommen, von Scheidung über Pensionierung bis hin zu Autounfällen. Damit sind 80 Millionen Bundesbürger behandelbar. Nimmt man dann noch prophylaktische Indikationen hinzu, von Stressmanagement und Burn-out-Prophylaxe bis hin zur Förderung eines gesund erhaltenden Lebensstils (Qui-gong-Kurse der Krankenkassen), dann ist jeder Einwohner der Bundesrepublik Deutschland gleich mehrfach psychotherapeutisch behandelbar. Diese Überlegung, die durch epidemiologische und fachliche Daten untermauert ist, zeigt, dass es nicht möglich ist, den Bedarf an Psychotherapie über eine Bedürfnisfeststellung zu klären. In der Bundesrepublik Deutschland gilt bereits jetzt, dass die Gruppe der psychotherapeutisch tätigen Ärzte und
Psychologen nach den Hausärzten die zweitgrößte Versorgungsgruppe im System ist und es bedarf keiner großen versorgungsepidemiologischen Kenntnisse, um sagen zu können, dass eine wesentliche Ausweitung der Psychotherapeutenzahlen im deutschen Gesundheitswesen nicht mehr realisierbar ist. Die Versorgung mit Psychotherapeuten in Deutschland hat einen Grad erreicht, dass man dieses System im internationalen Kontext fast schon nicht mehr verständlich machen kann.

Wenn also gilt, dass es auf der einen Seite eine begrenzte Zahl von Psychotherapeuten gibt (ca. 50000 bzw. etwa einer pro 1500 Einwohner) und auf der anderen Seite eine potenziell unbegrenzte Zahl von möglichen Behandlungsfällen, dann stellt sich in Ergänzung zu den Wirtschaftlichkeitsprüfproblemen die Zuweisungssteuerung bzw. «Ressourcenallokation» als weitere gutachterliche Aufgabe; d.h. die verfügbaren Therapieplätze werden so genutzt, dass die Patienten mit den besten Behandlungsergebnissen bevorzugt behandelt werden. Dabei ist zu berücksichtigen, dass das Gutachterverfahren bislang nicht kostensparend, sondern nur ressourcenallokativ wirksam ist. Wenn ein Therapeut nach gutachterlicher Ablehnung den Patienten A nicht behandelt, nimmt er in der dadurch frei werdenden Stunde eben Patient B in Therapie. Das Gutachterverfahren dient also ausschließlich dazu, den kostbaren Therapieplatz dem Patienten B anstatt dem Patienten A zugänglich zu machen. Die Frage ist, wie das Problem der Zuweisungssteuerung bzw. der Ressourcenallokation und letztlich der Sicherstellung notwendiger Behandlungen gelöst werden kann. Eine mögliche Vorgehensweise wäre, eine Psychotherapie erst nach Überweisung durch einen Facharzt oder Hausarzt aufzunehmen. Das mag unter inhaltlichen Aspekten sicherlich überlegenswert sein, gerade im ländlichen Raum sind aber jetzt schon bei niedergelassenen Nervenfachärzten oder psychosomatischen Medizinern (die ja selbst auch gleichzeitig Psychotherapeuten sind) lange Wartezeiten zu verzeichnen; angesichts der niedrigen Absolventenzahl einer Facharztausbildung in den medizinischen «P-Fächern» ist auch nicht abzusehen, dass hier zumindest in mittelfristiger Zukunft eine deutliche Verbesserung eintreten wird. Hier wird also lediglich die Wartezeit für eine Behandlung auf einen anderen Leistungsbringer verschoben. $\mathrm{Ob}$ eine gewünschte Patientenauswahl im Sinne von psychotherapeutisch behandlungsbedürftigeren Patienten stattfindet, muss jedoch bezweifelt werden.

Das Gutachterverfahren hat sich insgesamt bewährt und mögliche Schwachstellen lassen sich im Rahmen der bisherigen Verfahrenswege zeitnah ausbessern und ergänzen. Deshalb plädieren wir dafür, das Gutachterverfahren in einer inhaltlich (aber nicht durch zusätzliche psychometrische Verfahren) ergänzten (modifizierten) Form beizubehalten.

Rolf Meermann; Eberhard Okon, Bad Pyrmont

\section{Literatur}

Borgart E-J, Okon E, Meermann R: Sozialmedizinische Aspekte im Rahmen ambulanter Fachpsychotherapie. Forum Psychotherapeutische Praxis 2007;7:29-36. 